\title{
Detection and identification of loss of efficiency faults of flight actuators*
}

\author{
Daniel Ossmann ${ }^{1}$ and Andreas Varga ${ }^{2}$
}

\begin{abstract}
In this paper we apply model-based synthesis methodologies for the development of robust fault detection and diagnosis (FDD) systems for the loss of efficiency (LOE) type of faults of flight actuators. Typical LOE faults can be modeled as either additive or multiplicative faults. For additive LOE fault robust fault detection techniques in conjunction with signal processing based fault identification is used to design a robust FDD system. For the detection of multiplicative LOE faults model detection techniques are employed, which allows to simultaneously perform fault identification. The developed FDD systems have been tested in a full nonlinear aircraft simulation model to validate its detection and identification characteristics under realistic conditions.
\end{abstract}

\section{INTRODUCTION}

The detection and identification of a loss of efficiency (LOE) fault for a flight control surface actuator has been intensively studied (e.g., see [1], [2], [3] and references cited therein). The early detection of a LOE is important to prevent undesired effects on the flight mechanics, aircraft stability and fuel consumption. Two main types of LOEs are relevant for practical applications. A first type of LOE is caused by an unmeasurable change in the actuator's input signal, caused, for example, by incorrect signal transmissions from the flight control computer to the actuator or induction of an undesired current by external effects. The result is a corrupted inputoutput transfer gain [4], which can be assimilated with an additive input fault. A second type of LOE can be caused by a leakage within the actuator, or by an occurrence of a stall load, or by the disconnection of the actuator due to a brocken rod, or a damage of the control surface. The last two cases lead to increased bandwidth of the actuator due to the reduced aerodynamic loads, while first two cause a decrease of the bandwidth. This is due to a reduced available pressure in case of leakage and a convergency of the size of the acting aerodynamic force and the size of the force delivered by the actuator in case of the stall load. The type of LOE faults changing the actuators bandwidth can be assimilated with parametric (or multiplicative) faults. For the development of a fault tolerant flight control system, the robust detection and identification of LOE faults over the whole flight envelope, for different pilot maneuvers and wind conditions, and over

\footnotetext{
*This work was performed in the framework of the FP7 RECONFIGURE Project: Grant agreement AAT-2012-RTD-2314544.

${ }^{1}$ Resarch Associate, Department of Aircraft systems dynamics, Institute of System Dynamics and Control, DLR Oberpfaffenhofen, D-82234 Wessling, Germany; daniel.ossmann@dlr.de

${ }^{2}$ Senior Scientist, Department of Aircraft systems dynamics, Institute of System Dynamics and Control, DLR Oberpfaffenhofen, D-82234 Wessling, Germany; andreas.varga@dlr.de
}

the whole range of uncertain parameter variations is an important prerequisite.

In literature barely no approaches can be found explaining the detailed physical reasons for the possible LOE types of faults on electro-hydraulic actuators nor trying to detect and clearly identify them while respecting the mutual influence in the fault diagnosis process. This paper aims to close these gaps. Therefore, in this paper we address the problem of detecting and identifying the two types of LOE faults by proposing a complete methodology for the design of a dedicated model based fault detection and diagnosis (FDD) system. The main factors which contributed to a satisfactory solution of the LOE identification problems are: (1) accurate modeling of the fault-free and faulty actuators via simple linear parameter-varying (LPV) models; (2) an improved model based fault detection combined with signal based identification techniques; (3) the integrated tuning of the free parameters of the FDD system using multi-objective optimization techniques. In what follows, we describe more in detail the above aspects and apply the proposed methodology for the detection of LOE in the case of an aileron failure.

\section{THE FDD SYSTEM FOR LOE DETECTION AND IDENTIFICATION}

The FDD system for the monitoring of LOE faults is depicted in Fig. 1. For each type of LOE, a separate channel is used to detect and identify the respective type of fault. Besides a dedicated residual generator, each channel includes blocks for residual evaluation, decision making and fault identification (only for the additive case). The fault identification consists in the determination of the main characteristics of the LOE from the generated residual signal $r$ in the case of an additive fault, or the estimation of the gain of the faulty actuator (e.g., by detecting the corresponding fault model), in the case of a parametric fault. Both types of LOE faults can be only detected if a sustained control activity is provided.

The FDD system structure in Fig. 1 includes two residual generators for fault detection of additive faults or model detection in the case of a parametric fault. The residual generators process the commanded actuator position $u$ and the measured current actuator position $y$ and generate the scalar residual signal $r$ in the additive case and the residual vector $r_{m}$ in the parametric case. For the robust fault detection considered in this paper, LPV gain-scheduled detectors are used, where $\rho$ is the vector of scheduling variables as described in Section III. The residual evaluation blocks compute specific approximations $\theta$ or $\theta_{m}$ of the norms of the components of the residual signals. These values are 


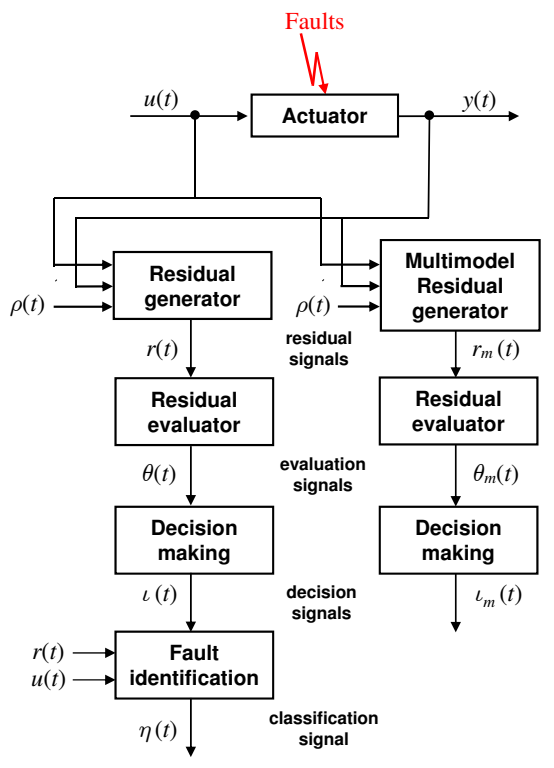

Fig. 1. FDD system for LOE monitoring

used in the decision making blocks. In the additive case, a threshold-based decision logic is employed to generate the decision signal $\iota$, which, if nonzero, triggers a specific signal based fault identification processes. The output of the fault identification block is the classification signals $\eta$, which indicates the presence or absence of an additive LOE fault. In the parametric case, the threshold-based decision logic determines the signature vector $\iota_{m}$, which codifies the detected fault model. In this case, the fault identification is implicitly performed.

The employed model-based methodology for the design of the elements of the FDD system in Fig. 1 has the following main steps:

1) Development of suitable synthesis models of the underlying actuator;

2) Synthesis of residual generators for robust fault detection;

3) Setting up of the residual evaluation and decision making blocks;

4) Development of signal processing based fault identification schemes;

In what follows we describe these main steps and present an application of the proposed LOE identification techniques in the case of a civil aircraft elevator.

\section{LPV MODEL GENERATION FOR THE NONLINEAR ACTUATOR MODEL}

In this section we describe the development of a quasiLPV approximate model for a system formed from an actuator and the associated control surface. For the details on the derivation of the quasi-LPV model see [5]. The resulting actuator model has a first order LPV-system representation of the form

$$
\dot{x}=k(\rho)(u-x)
$$

with the output $y=x$, where $x$ and $y$ are the rod position and $u$ is the commanded position. The gain $k(\rho)$ generally depends on both measurable and unmeasurable parameters contained in a vector $\rho$.

For the actuator we employ a simplified nonlinear dynamic model of a hydraulic servo controlled actuator described by a first order nonlinear state equation of the form [2]

$$
\dot{x}=K(p, x, \dot{x})(u-x)
$$

with

$$
K(p, x, \dot{x}):=K_{c i} K_{p} \sqrt{\frac{\Delta P(x)-\frac{F_{a e r o}(p, x, \dot{x})+K_{d} \dot{x}^{2}}{S}}{\Delta P_{\text {ref }}}}
$$

where $K_{p}$ is the servo control gain, $K_{c i}$ is a gain to convert an estimated current to a corresponding rod speed, $\Delta P$ is the hydraulic pressure delivered to the actuator, $\Delta P_{\text {ref }}$ is a differential pressure for a fully opened servo-valve (maximum rod speed), $F_{\text {aero }}$ represents the aerodynamic forces at the control surface, $K_{d} \dot{x}^{2}$ represents the estimated servo-control load of the adjacent actuator in damping mode and $S$ is the actuator piston surface area. The components of the vector $p$ are the calibrated airspeed $V_{\text {cas }}$, the aircraft altitude $h$ and the angle of attack $\alpha$.

To get a simple quasi-LPV model approximating (2) with good accuracy, we approximated the nonlinear gain (3) by an easily computable gain $k(p, x, \dot{x})$, which is then used in the first order actuator model as given in (1). The main variations of $K$ are caused by the aerodynamic force $F_{\text {aero }}$ that acts on the control surface, where $F_{\text {aero }}$ itself usually depends on the parameters in $p$, the actuator position $x$ and the sign of the actuator position rate $\dot{x}$. To develop a simpler version of this nonlinear gain (3), a Taylor-series is developed, leading to the form

$$
\begin{aligned}
& k(p, x, \dot{x})= \\
& C_{0}(p)+C_{1}(p) \operatorname{sign}(\dot{x})+x\left(C_{2}(p)+C_{3}(p) \operatorname{sign}(\dot{x})\right),
\end{aligned}
$$

where for fixed $p, C_{0}(p)$ can be interpreted as the nominal gain, $C_{3}(p)$ describes the influence of the deflection angle $x$ on $k$, while the factor $\operatorname{sign}(\dot{x})$ allows to distinguish between upward and downward movements of the control surface.

The chosen functional dependence on $x$ and $\operatorname{sign}(\dot{x})$ reflects the actual behavior of the actuator dynamics for different control surface positions and signs of deflection rate. For $C_{i}(p), i=0,1,2,3$, affine approximations have been used, where the intervening constant coefficients have been determined using parameter fitting techniques based on comparing the output responses of the nonlinear actuator model (2) and LPV-model (1). The final form of $k(\rho)$, with $\rho=(x, \operatorname{sign}(\dot{x}), p)$ is simple enough to be used in LPVmodel based fault diagnosis applications.

\section{DETECTION AND IDENTIFICATION OF ADDITIVE LOES}

\section{A. LPV residual generator for detection of additive LOEs}

Assume temporarily that the parameters in $\rho$ are constant. In this case we can use an input-output representation of the 
actuator fault model in the form

$$
\mathbf{y}(s)=G_{u}(s, \rho) \mathbf{u}(s)+G_{f}(s, \rho) \mathbf{f}(s),
$$

where $\mathbf{y}(s), \mathbf{u}(s)$, and $\mathbf{f}(s)$ are the Laplace-transformed quantities of $y(t), u(t)$, and $f(t)$, respectively, where the additive fault signal can be defined as $f(t):=\left(1-\nu_{a}\right) u(t)$. In (5), $G_{u}(s, \rho)$ and $G_{f}(s, \rho)$ are parameter dependent transfer functions corresponding to (1)

$$
G_{u}(s, \rho)=\frac{k(\rho)}{s+k(\rho)}, \quad G_{f}(s, \rho)=\left(1-\nu_{a}\right) G_{u}(s, \rho)
$$

The value $\nu_{a}=1$ corresponds to the fault-free case, while $0<\nu_{a}<1$ corresponds to a LOE fault. In the case of the aileron actuator model we use $\rho=\left(x, \operatorname{sign}(\dot{x}), V_{\text {cas }}, h, \alpha\right)$.

As residual generator we use a parameter dependent filter of the form

$$
\mathbf{r}(s)=Q(s, \rho)\left[\begin{array}{l}
\mathbf{y}(s) \\
\mathbf{u}(s)
\end{array}\right]
$$

where $Q(s, \rho)$ is the $1 \times 2$ transfer-function matrix of the filter, which explicitly depends on the measurable parameter $\rho$ (e.g., via an equivalent state-space realization of the filter). For a physically realizable filter, $Q(s, \rho)$ must be robustly stable, having only poles with negative real parts for all values of $\rho$. The robust fault detection synthesis problem addresses the robustness of the fault detection system with respect to the measurable parameter $\rho$ by attempting to achieve robustness using an LPV gain scheduling approach.

To address the robust detection of LOE, we employed the synthesis method described in [5]. Accordingly, we can use a first order detector of the form

$$
Q(s, \rho)=\left[\begin{array}{ll}
\frac{a}{k_{0}} \frac{s+k(\rho)}{s+a} & -\frac{k(\rho) a}{k_{0}(s+a)}
\end{array}\right],
$$

where $a$ is an arbitrary positive value specifying the dynamics of the detector and $k_{0}$ is a typical nominal value of the gain $k(\rho)$. By replacing in (7) $\mathbf{y}(s)$ by its expression in (5), we obtain the internal form of the detector

$$
\mathbf{r}(s)=R_{u}(s, \rho) \mathbf{u}(s)+R_{f}(s, \rho) \mathbf{f}(s)
$$

where

$$
\begin{aligned}
& {\left[R_{u}(s, \rho) \mid R_{f}(s, \rho)\right]:=} \\
& Q(s, \rho)\left[\begin{array}{c|c}
G_{u}(s, \rho) & G_{f}(s, \rho) \\
1 & 0
\end{array}\right]
\end{aligned}
$$

The choice (8) of $Q(s, \rho)$ guarantees an exact decoupling of control inputs in (10), thus $R_{u}(s, \rho)=0$. The corresponding fault-to-residual transfer function is

$$
R_{f}(s, \rho)=\left(1-\nu_{a}\right) \frac{k(\rho)}{k_{0}} \frac{a}{s+a}
$$

Thus, as long as $f=\left(1-\nu_{a}\right) u \neq 0$, the additively formulated LOE fault can be easily detected.

The LPV state-space realization of the residual generator (7) can be always obtained in the form

$$
\begin{aligned}
\dot{x}_{Q}(t) & =A_{Q} x_{Q}(t)+B_{Q}(\rho)\left[\begin{array}{l}
y(t) \\
u(t)
\end{array}\right] \\
r(t) & =C_{Q} x_{Q}(t)+D_{Q}(\rho)\left[\begin{array}{l}
y(t) \\
u(t)
\end{array}\right]
\end{aligned}
$$

For the detector (8), the state-space matrices are

$$
\begin{array}{ll}
A_{Q}=-a, & B_{Q}(\rho)=a\left[\begin{array}{ll}
\frac{k(\rho)-a}{k_{0}} & \frac{k(\rho)}{k_{0}}
\end{array}\right], \\
C_{Q}=1, & D_{Q}=\left[\begin{array}{ll}
\frac{a}{k_{0}} & 0
\end{array}\right] .
\end{array}
$$

The chosen form (8) of the detection filter leads to a statespace realization with a constant feed-through matrix $D_{Q}$. This has the major advantage to prevent all direct effects on $r$ of the discontinuities in the scheduling signal $\rho$ (e.g., jumps due to the presence of the signum-function in (4)).

\section{B. Residual evaluation}

The evaluation of the residual signals often requires the computation of a measure of the residual signal energy, for which the 2-norm of the signal is usually an appropriate choice. For this purpose, the so-called Narendra signal evaluation scheme can be used of the form

$$
\theta(t)=\alpha|r(t)|+\beta \int_{0}^{t} e^{-\gamma(t-\tau)}|r(\tau)| d \tau,
$$

where $\theta(t)$ can be generated by the first order differential equation

$$
\begin{aligned}
& \dot{\xi}(t)=-\gamma \xi(t)+\beta|r(t)| \\
& \theta(t)=\xi(t)+\alpha|r(t)|,
\end{aligned}
$$

The filter parameters $\alpha \geq 0$ and $\beta \geq 0$ are suitable weights for instantaneous and long-term values, respectively, while $\gamma>0$ is the forgetting factor.

\section{Decision making}

The evaluation signal $\theta(t)$ is compared to a specific threshold $\tau$ in the decision making process to determine the decision signal $\iota(t)$ using the decision logic

$$
\iota(t)= \begin{cases}1, & \text { if } \theta(t) \geq \tau \\ 0 & \text { otherwise }\end{cases}
$$

The signal $\theta(t)$ is ideally equal to zero or sufficiently small in fault free situations, whereas it shall exceed the threshold $\tau$ when a fault occurs in the system. Hence, the appropriate selection of the values of the free parameters $\alpha$, $\beta$ or $\gamma$, together with an appropriate threshold $\tau$ essentially influences the performance of the FDD system.

\section{Determination of the detection threshold}

The free parameters $\alpha, \beta$ and $\gamma$ of the residual evaluation blocks and the threshold $\tau$ used in the decision blocks must be chosen to ensure that the requirements regarding typical performance criteria used in the industry as the false alarm rate (FAR), the missed detection rate (MDR) or the detection time performance (DTP) are fulfilled. The simultaneous minimization of these quantities (e.g., by using multi-objective optimization techniques) would provide the best achievable detection performance. Unfortunately, analytical expressions of FAR and MDR are not available, and only surrogates can be used which are suitable only when we require FAR $=0$ and $\mathrm{MDR}=0$. The applicability of such surrogates based tuning has been demonstrated in [5]. 
For an optimization based tuning setup, the requirements for the lack of false alarms and missed detections can be formulated as either optimization criteria or constraints. In the absence of faults, the requirement for no false alarms leads to a constraint on the false alarm bound

$$
\tau_{f}:=\sup _{f=0} \theta(t)<\tau,
$$

where the supremum is taken for all admissible operation points, all relevant aircraft maneuvers, all admissible variations of uncertain parameters and for all relevant disturbances.

The requirement for no missed detection can be also expressed as a constraint on the detection bound

$$
\tau_{d}:=\inf _{t \in\left[t_{f}, t_{\text {detec }}\right]} \theta(t) \geq \tau,
$$

which must be satisfied for all relevant fault situations. Here, $t_{\text {detec }}$ is the maximum admissible fault detection time and $t_{f}$ is the fault occurrence time. To ensure simultaneously the lack of false alarms and of missed detections, the condition $\tau_{f}<\tau_{d}$ must be fulfilled. Different optimization strategies are presented in [6].

\section{E. Fault identification}

Starting from the detailed actuator description (2), we can assume that if the additive LOE occurs the behavior of the actuator can be described by

$$
\dot{\hat{x}}=K(p, \hat{x}, \dot{\hat{x}})\left(\nu_{a} u-\hat{x}\right)
$$

with $\hat{y}=\hat{x}$, where we assume $\nu_{a}$ is a constant parameter which describes the loss of efficiency of the actuator. The output values $y$ of the fault free actuator can be generated using (1), as an LPV reference model. It follows that the outputs of the faulty actuator $\hat{y}(t)$ and the fault free actuator model $y(t)$ are related at all time moments simply as $\hat{y}(t)=$ $\nu_{a} y(t)$. Thus, the time-dependent ratio of the two outputs

$$
\hat{\nu}_{a}(t)=\frac{\hat{y}(t)}{y(t)} .
$$

can be used to check the assumption of constant scaling $\nu_{a}$.

Let $n$ be a given number of samples, $T$ a given sampling period, $t_{d}$ the fault detection time, and $\hat{\nu}_{a, k}:=\hat{\nu}_{a}\left(t_{d}+(k-\right.$ 1)T). The variance $\sigma_{\hat{\nu}_{a}}$ of $\hat{\nu}_{a}$ can be determined in real-time using the numerically stable recursive algorithm [8]

$$
\begin{aligned}
& m_{k}=m_{k-1}+\left(\hat{\nu}_{a, k}-m_{k-1}\right) / k \\
& s_{k}=s_{k-1}+\left(\hat{\nu}_{a, k}-m_{k-1}\right)\left(\hat{\nu}_{a, k}-m_{k}\right)
\end{aligned}
$$

for $k=2, \ldots, n$, where $m_{1}=\hat{\nu}_{a, 1}$ and $s_{1}=0$. Then, $\sigma_{\hat{\nu}_{a}}=s_{n} /(n-1)$ and the LOE is confirmed if $\sigma_{\hat{\nu}_{a}} \leq \tau_{\sigma_{\nu_{a}}}$, where $\tau_{\sigma_{\nu a}}$ is a suitable threshold for zero variance. Typical values of the main parameters of the identification procedure are $T^{\prime}=0.01 \mathrm{~s}$ and $n=50 \div 300$. The confirmation signal of the LOE fault can be set as

$$
\eta= \begin{cases}1 & \text { if }\left(\|u\|>\tau_{u}\right) \wedge\left(\sigma_{\hat{\nu}_{a}}<\tau_{\sigma_{\nu_{a}}}\right) \\ 0 & \text { otherwise. }\end{cases}
$$

where $\tau_{u}$ is a threshold for nonzero inputs. For the computation of $\|u\|$ the Narendra-filtering scheme (13) can be employed.

\section{DETECTION AND IDENTIFICATION OF PARAMETRIC LOES}

Several LOE actuator faults like leakage in the hydraulic system, stall load, broken actuator rod or surface damage lead to changes in the actuator gain (3) and thus can be interpreted as parametric faults. Due to low fault sensitivities (only during transients), the detection of such actuator faults using methods for additive faults is often difficult, while the fault identification is often questionable. In this section we describe an alternative approach to simultaneously achieve the fault detection and identification using an approach based on model detection techniques.

\section{A. Multimodel residual generator for detection of parametric LOES}

The actuator gain (3) of the fault-free actuator can be modified to express the effects of faults in the form

$K_{f}(p, x, \dot{x}):=K_{c i} K_{p} \sqrt{\frac{\nu_{p, 1} \Delta P(x)-\frac{\nu_{p, 2} F_{a e r o}(p, x, \dot{x})+K_{d} \dot{x}^{2}}{S}}{\Delta P_{\text {ref }}}}$,

where $0<\nu_{p, 1} \leq 1$ and $0 \leq \nu_{p, 2} \leq 1$ are factors used to describe the effects of the parametric changes due to leakage or surface damage/disconnection, respectively. To account for gradual degradation of actuator performance (i.e., different degrees of LOE), consider $N+1$ distinct values $\left(\nu_{p, 1}^{(i)}, \nu_{p, 2}^{(i)}\right)$, for $i=0,1, \ldots, N$, of the pair $\left(\nu_{p, 1}, \nu_{p, 2}\right)$, where $\left(\nu_{p, 1}^{(0)}, \nu_{p, 2}^{(0)}\right):=(1,1)$ corresponds to the fault free case. An exception is the stall load, as it always occurs, if $\nu_{p, 1} \Delta P(x) S \approx \nu_{p, 2} F_{\text {aero }}(p, x, \dot{x})+K_{d} \dot{x}^{2}$ in (21). However, also this case, which cannot be explicitly modeled using $\nu_{p, 1}$ and $\nu_{p, 2}$, is classified as faulty behavior, which has to be detected and identified. This is achieved by a distinct modeling of the very slow actuator dynamics in case of a stall load, while the its effect is not considered in the modeling of the fault free behavior. Note that the effect of leakage in the system supports the occurrence of a stall load, as the available pressure in the system is decreased.

Let $k^{(i)}(\rho)$ be a parametric approximation of the gain (21) of the form (4), obtained for $\left(\nu_{p, 1}, \nu_{p, 2}\right)=\left(\nu_{p, 1}^{(i)}, \nu_{p, 2}^{(i)}\right)$. Assume that we employ $N+1$ parametric models of the form

$$
\mathbf{y}^{(i)}(s)=G_{u}^{(i)}(s, \rho) \mathbf{u}(s)
$$

for $i=0,1, \ldots, N$, where

$$
G_{u}^{(i)}(s, \rho)=\frac{k^{(i)}(\rho)}{s+k^{(i)}(\rho)}
$$

The model detection problem can be formulated as follows [7]: Determine a batch of $N+1$ filters with scalar outputs of the form

$$
\mathbf{r}^{(i)}(s)=Q^{(i)}(s, \rho)\left[\begin{array}{l}
\mathbf{z}(s) \\
\mathbf{u}(s)
\end{array}\right], i=0,1, \ldots, N
$$

such that for all $u(t)$ we have:

(i) $\quad r^{(i)}(t)=0$ when $z(t)=y^{(i)}(t)$;

(ii) $\quad r^{(i)}(t) \neq 0$ when $z(t)=y^{(j)}(t)$ for $i \neq j$;

(iii) $r^{(i)}(t)$ is asymptotically bounded. 
To fulfill condition $(i)$ we choose

$$
Q^{(i)}(s, \rho)=\left[\begin{array}{cc}
1 & -G_{u}^{(i)}(s, \rho)
\end{array}\right], i=0,1, \ldots, N
$$

which is by construction stable, so condition (iii) is automatically fulfilled. Furthermore, we have for $z(t)=y^{(j)}(t)$ and for $j \neq i$

$\mathbf{r}^{(i)}(s)=Q^{(i)}(s, \rho)\left[\begin{array}{l}\mathbf{y}^{(j)}(s) \\ \mathbf{u}(s)\end{array}\right]=\left(G_{u}^{(j)}(s, \rho)-G_{u}^{(i)}(s, \rho)\right) \mathbf{u}(s)$

which shows that condition $(i i)$ is also fulfilled, provided $u(t)$ is nonzero. Due to the presence of a zero in the origin of $G_{u}^{(j)}(s, \rho)-G_{u}^{(i)}(s, \rho), u$ must be also nonconstant.

The overall residual generator used for model detection results in the form

$$
\mathbf{r}_{m}(s)=Q_{m}(s, \rho)\left[\begin{array}{l}
\mathbf{y}(s) \\
\mathbf{u}(s)
\end{array}\right]
$$

where

$$
\mathbf{r}_{m}(s):=\left[\begin{array}{c}
\mathbf{r}^{(0)}(s) \\
\mathbf{r}^{(1)}(s) \\
\vdots \\
\mathbf{r}^{(N)}(s)
\end{array}\right], \quad Q_{m}(s, \rho):=\left[\begin{array}{c}
Q_{m}^{(0)}(s, \rho) \\
Q_{m}^{(1)}(s, \rho) \\
\vdots \\
Q_{m}^{(N)}(s, \rho)
\end{array}\right]
$$

\section{B. Residual evaluation and decision making}

For the detection of the $i$-th model, all components of the residual vector $r_{m}(t)$ must be nonzero, excepting the $i$-th component. For each component $r^{(i)}(t)$ of the $N+1$ dimensional residual vector $r_{m}(t)$ an evaluation signal $\theta^{(i)}(t)$ of the form (13) can be generated to build the evaluation vector

$$
\theta_{m}(t):=\left[\theta^{(0)}(t), \ldots, \theta^{(N)}(t)\right]^{T}
$$

and define the decision vector $\iota_{m}$ as

$$
\iota_{m}(t):=\left[\iota^{(0)}(t), \ldots, \iota^{(N)}(t)\right]^{T}
$$

whose $i$-th component is defined as

$$
\iota^{(i)}(t)= \begin{cases}1, & \text { if } \theta^{(i)} \geq \tau^{(i)}\|u\|_{2} \\ 0 & \text { otherwise. }\end{cases}
$$

where $\tau^{(i)}$ is a suitable threshold for nonzero gains. For the threshold selection we can exploit (27) and choose

$$
\tau^{(i)}:=\inf _{j \neq i ; \rho}\left\|G_{u}^{(j)}(s, \rho)-G_{u}^{(i)}(s, \rho)\right\|_{\infty}
$$

Furthermore, with suitable scalings of the filters (26), it is possible to use a unique value, say $\tau_{m}$, for all thresholds $\tau^{(i)}$.

Regarding the decision making, note that the only valid signature vectors for model detection are those with a single zero component. Therefore, all other signatures, as for example, all components nonzero or all components zero rule out cases when there is no LOE fault or the detection conditions $u \neq 0$ or $u$ nonconstant are not fulfilled.

\section{APPLICATION EXAMPLE}

In this section we describe the application of the methodology described in this paper to the detection LOE faults of an aileron surface controlled by an electro-hydraulic actuator. The nonlinear actuator model of the aileron is part of a nonlinear model of a closed-loop aircraft including a nonlinear control law ensuring robust stability over the whole flight envelope. For tuning purposes, the closed-loop aircraft model has been augmented with an FDD system as in Fig. 1, which includes the LPV residual generator for additive fault detection and the multi-model based residual generator for parametric fault detection.

\section{A. Additive $L O E$}

Fig. 2 shows the input and output signals of the actuator before and after the occurrence of a LOE fault. In the first 15 seconds the output signal $y$ follows the commanded signal $u$ almost instantly. At $t_{f}=15 \mathrm{~s}$ an additive LOE of $20 \%$ occurs, leading to a significant difference between the two signals. To avoid false alarms and missed detections, an optimizationbased tuning of the values of the parameters $(\alpha, \beta, \gamma)$ of the residual evaluation block and the threshold $\tau$ of the decision making block has been done by maximizing the detection gap $\tau^{d}-\tau^{f}$ [6]. The optimal setting with $\alpha=0, \beta=1$, $\gamma=0.2$ and $\tau=0.1$ leads to a completely satisfactory fault detection performance. Fig. 3 shows the detection times for four input amplitudes $\|u\|_{\infty}$ (defined in terms of the maximum allowed amplitude $u_{\max }$ ), for different values of the additive efficiency loss up to $90 \%$. The results show that higher degradations of the actuator effectiveness lead to shorter detection times. Furthermore, as it is to be expected, if the input amplitude is decreasing, the detection of the faults becomes slower and there exists a well defined least detectable fault amplitude (see the dash-dotted line in Fig. 3). For fault identification, $n=50$ additional values of the outputs (faulty and fault-free) have been collected using a sampling period of $T=0.01 \mathrm{~s}$. This led to quit fast confirmation times of LOE of about $0.5 \mathrm{~s}$.

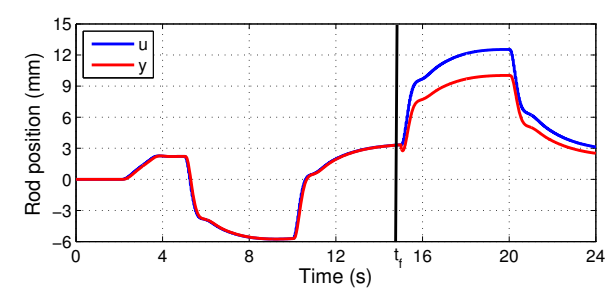

Fig. 2. Effect of the additive LOE

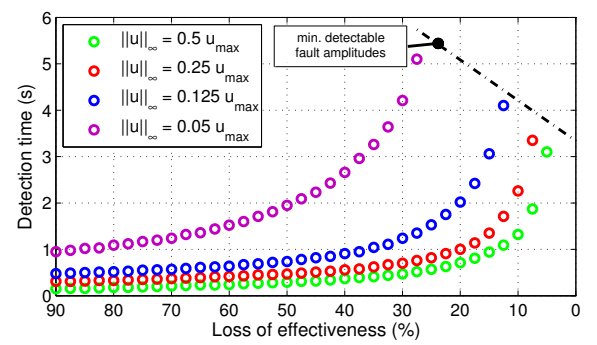

Fig. 3. Detection times for different magnitudes of input and failure 


\section{B. Parametric $L O E$}

Fig. 4 shows the output signals of the fault-free actuator and of a faulty actuator with a loss of the hydraulic pressure of $25 \%$ occurring at $15 \mathrm{~s}$. As only the dynamical behavior of the actuator is influenced, there is only a small difference in the two signals, making the parametric LOE fault nearly undetectable using additive fault based approaches. This is the reason why the model detection based approach has been used for the detection and identification of this type of LOE fault. For the case of parametric LOE fault, we employed six

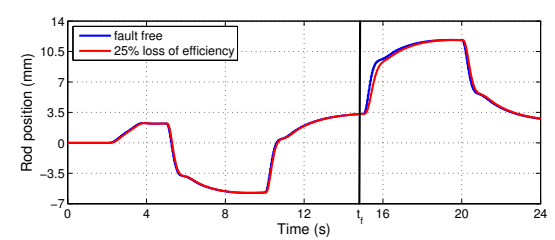

Fig. 4. Effect of the parametric LOE

actuator models for the following pairs of fault parameters: $\left(\nu_{p, 1}^{(0)}, \nu_{p, 2}^{(0)}\right):=(1,1)$ for the fault free case, $\left(\nu_{p, 1}^{(1)}, \nu_{p, 2}^{(1)}\right):=$ $(1,0),\left(\nu_{p, 1}^{(2)}, \nu_{p, 2}^{(2)}\right):=(1,0.33)$, and $\left(\nu_{p, 1}^{(3)}, \nu_{p, 2}^{(3)}\right):=(1,0.66)$, for $100 \%, 66 \%$ and $33 \%$ loss of the control surface effectiveness, respectively, and $\left(\nu_{p, 1}^{(4)}, \nu_{p, 2}^{(4)}\right):=(0.85,1)$, for a $15 \%$ loss of the hydraulic pressure. Additionally, to describe the situation when $\Delta P(x) S \approx F_{\text {aero }}(p, x, \dot{x})+K_{d} \dot{x}^{2}$ holds, a constant first order linear model is employed. Correspondingly, approximations of the actuator gains $k^{(i)}(\rho)$ of the form (4) have been determined using parameter fitting techniques described in Section III. For the special case of $100 \%$ LOE due to either actuator disconnection or a total surface damage, the following easily implementable exact gain can be alternatively used

$$
k^{(1)}(\rho)=K_{c i} K_{p} \sqrt{\frac{\Delta P(x)}{\Delta P_{r e f}}}
$$

For the case of a stall load the constant gain $k^{(5)}(\rho)=0.1$, describing the decreased actuator dynamics during a stall load, is employed. Note, that the effects decreasing the actuator bandwidth are not considered in the fault free model, to be able to clearly identify the stall load. The six actuator models of the form (23) have been used to define the corresponding six scalar output detectors (26), which enter in the overall detector (28). For all six Narendra filters in the evaluation block, the same values of the parameters have been used, namely $\alpha=0, \beta=1, \gamma=0.1$. Using suitable scaling of the individual detectors, the same detection threshold value of $\tau_{m}=0.15$ has been used for all $\tau^{(i)}$.

Fig. 5 shows the results of the model detection for degradations of the control surface between $0 \%$ and $100 \%$ as well as for leakage in the pressure supply of up to $20 \%$. The maximum leakage value corresponds to an appearing stall load, as the available pressure is too small to move the surface against the acting aerodynamic force. The red vertical lines indicate the grid values corresponding to the faults for which a LPV model has been developed and used in the detector. The blue circles show the results of the model identification obtained using the closed-loop flight control system augmented with the developed FDD system. Important to notice is that all nearby models to a grid value have been correctly detected (i.e., there are no switching between models) and therefore no false classification of the fault occurred. A more accurate fault identification can be obtained by increasing the number of models (with the associated increased computational load).

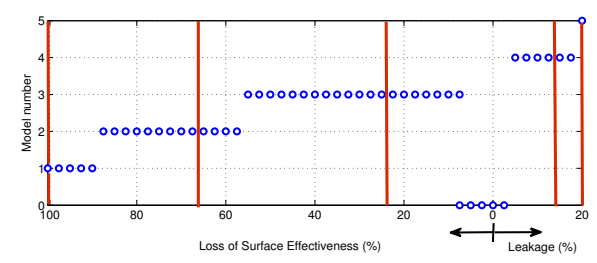

Fig. 5. Detected model during fault simulations

\section{CONCLUSIONS}

In this paper we proposed a model-based synthesis and tuning methodology for the development of an FDD system for the detection and identification of loss of efficiency failure cases for aircraft actuators. The main features of the proposed approach which confers superior performance over existing approaches are: (1) relying on accurate LPV models allowing the synthesis of robust fault detection filters, (2) application of advanced synthesis methods of LPV residual generators guaranteeing robust fault detection, (3) employing integrated optimization-based tuning of the parameters of the fault evaluation and decision making blocks, including the determination of detection thresholds to guarantee no false alarms and no missed detections, (4) employing real-time implementable fault identification method. The proposed methodology has been successfully applied for the design of an FDD system for the identification of the additive and parametric LOE faults of an aileron actuator.

\section{REFERENCES}

[1] A. K. Caglayan, K. Rahnamai, and S.M. Allen, Detection, identification and estimaton of surface damage/actuators failure for high performance aircraft. In Proc. American Control Conference, 1988, 2206-2212.

[2] L. Marton and D. Ossmann, Energetic approach for control surface disconnection fault detection in hydraulic aircraft actuators. In Proc. SAFEPROCESS12, Mexico City, 2012.

[3] L. Van Eykeren, Q. Chu and J.A. Mulder, Actuator fault detection by aerodynamic model identification. In Proc. SAFEPROCESS12, Mexico City, 2012.

[4] J. D. Boškovic and R.K. Mehra, A Multiple model-based reconfigurable flight control system design. In Proc. IEEE Conference on Decision and Control, Tampa, Florida, 1998.

[5] A. Varga, S. Hecker and D. Ossmann, Diagnosis of actuator faults using LPV-gain scheduling techniques. In Proc. of AIAA Guidance, Navigation, and Control Conference, Portland, Oregon, USA, 2012.

[6] A. Varga and D. Ossmann, LPV-techniques based robust diagnosis of flight actuator faults. Control Engineering Practice (submitted 2013).

[7] A. Varga, Least order fault and model detection using multi-models. In Proc. IEEE Conference on Decision and Control, Shanghai, China, 2009.

[8] B. P. Welford, Note on a method for calculating corrected sums of squares and products, Technometrics, vol. 4, 419-420, 1962. 